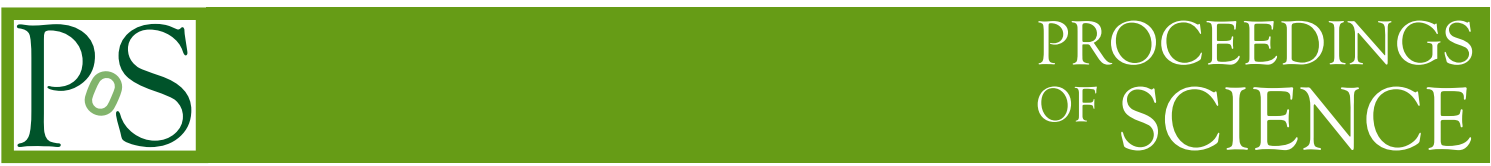

\title{
Low-power compact radio galaxies at high angular resolution
}

\author{
Marcello Giroletti ${ }^{* 1}$, Gabriele Giovannini ${ }^{1,2}$, and Greg B. Taylor ${ }^{3}$ \\ ${ }^{1}$ INAF Istituto di Radioastronomia, via Gobetti 101, 40129 Bologna, Italy \\ ${ }^{2}$ University of Bologna, Department of Astronomy, via Ranzani 1, 40127 Bologna, Italy \\ ${ }^{3}$ University of New Mexico, Department of Physics and Astronomy, Albuquerque, NM 87131, USA \\ E-mail: giroletti@ira.inaf.it, ggiovann@ira.inaf.it, gbtaylor@unm.edu
}

\begin{abstract}
The true nature of Low-Power Compact (LPC) radio sources is not well understood. They have similar power as FR I radio galaxies but smaller linear sizes. In this contribution, we exploit phase-referenced VLBI images of five such sources to discuss the properties of their nuclear regions, as well as 8- and 22-GHz VLA data to resolve the sub-arcsecond-scale resolution structures. Our data reveal flat-spectrum, compact cores (down to a few mJy level) in four of the five sources and the absolute astrometry provided by the phase referencing allows us to identify the centre of activity in the VLA images. Based on our results, we rule out the presence of strong relativistic effects in these LPCs, so they must be intrinsically small and possibly frustrated or short lived. Fits of continuous injection models yield spectral ages in the range $10^{5}-10^{7}$ yrs.
\end{abstract}

In memory of Prof. Luciano Pentimalli

8th European VLBI Network Symposium

September 26-29, 2006

Toruń, Poland

${ }^{*}$ Speaker. 


\section{Introduction}

Very high-resolution observations of low-power radio sources are becoming more and more feasible as the sensitivity of VLBI arrays increases. A review of the most interesting recent results in these area has been given by [1]. Here, we will focus on low-power compact (LPC) sources, i.e. sources that have radio powers similar to Fanaroff-Riley (FR) I radio galaxies $\left(<10^{25} \mathrm{~W} \mathrm{~Hz}^{-1}\right)$ but much smaller linear sizes (typically $<10 \mathrm{kpc}$ ). In particular, we are addressing sources from the Bologna Complete Sample (BCS, [2] ]), consisting of 95 radio sources with redshifts $z<0.1$. Eighty-one of them are classified as extended FR I or II radio galaxies, whereas the remaining 14 have compact kiloparsec-scale morphology. Except the BL Lacertae objects Mrk 421 and Mrk 501, whose compactness is due to projection, and a few other well-studied sources (e.g. 4C31.04, NGC 4278 [3, 田), the properties of the remaining LPCs are not known. It is not clear if they have to be ascribed to geometrical-relativistic effects, low age, frustration by a dense medium, as well as short-lived or intrinsically weak jet activity. In this paper (as well as in a more detailed work [5]), we make use of high-resolution radio images supplemented with spectra to clarify the true nature of LPCs. Throughout the paper, we adopt $\mathrm{H}_{0}=70 \mathrm{~km} \mathrm{sec}^{-1} \mathrm{Mpc}^{-1}, \Omega_{M}=0.3$ and $\Omega_{\Lambda}=0.7$.

\section{Observations}

VLBI observations have been carried out in phase-referenced mode using the VLBA at 1.6 GHz on 7 August 2003. The typical resolution is $\sim 5$ mas with natural weighting and we attain an rms noise of a few $\times 0.1 \mathrm{mJy} / \mathrm{beam}$. Applying solutions from the respective phase calibrators, we have successfully detected 4 of the 5 observed sources; we note that these are either new detections or great improvements over previous VLBI images of the same sources. Moreover, thanks to phase referencing, we have also obtained absolute position information that allows us to identify the locations of relevant milliarcsecond-resolution features in larger-scale VLA images. The VLA images have been taken in A configuration at 8 and $22 \mathrm{GHz}$, the latter providing HPBWs as small as $\sim 0.1^{\prime \prime}$, thus resolving the sub-arcsecond structure of the sources well.

\section{Results}

\subsection{VLA data: from blobs to jets}

In Fig. 1, we show a collection of images from the literature (top panels, [6]) and as obtained with our observations (bottom panels). While the older images detect only point-like or barely resolved components, our high-frequency data show that these sources have rich sub-structures resembling classical radio galaxies, both edge-dimmed $(0222+36,0258+35)$ and edge-brightened $(1037+30)$, and even a wide angle tail source $(1855+37)$. However, none of these radio sources has a linear size exceeding $10 \mathrm{kpc}$. Moreover, the cores are typically rather faint and account for only a small fraction of the total flux density. In the case of $1855+37$, the $22-\mathrm{GHz}$ observations failed to detect the source.

\subsection{VLBI data: $0222+36$}

This source at $z=0.0334\left(1^{\prime \prime}=0.66 \mathrm{kpc}\right)$ has a flux density of $337 \mathrm{mJy}$ at $408 \mathrm{MHz}$, corresponding to a monochromatic total power of $10^{23.9} \mathrm{~W} \mathrm{~Hz}^{-1}$. Our VLA observations resolved the 


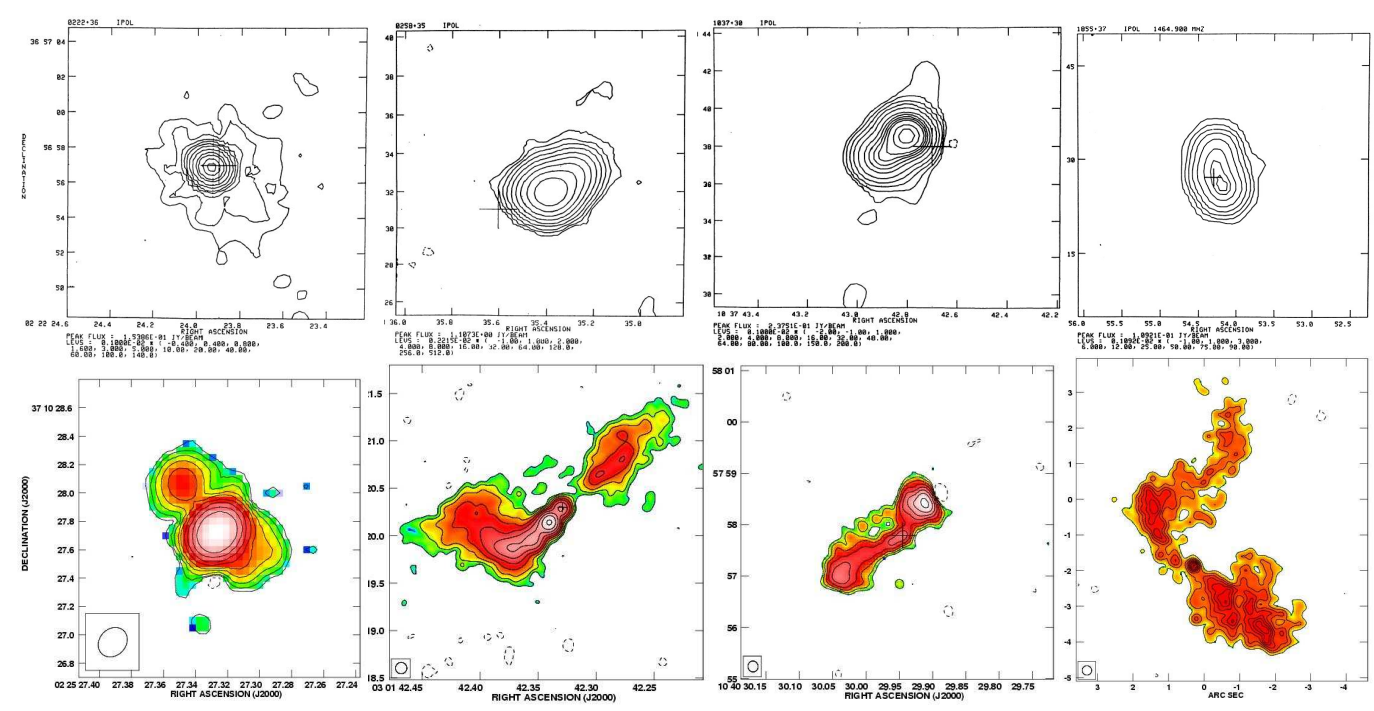

Figure 1: VLA images. Top: literature, bottom: new high frequency data
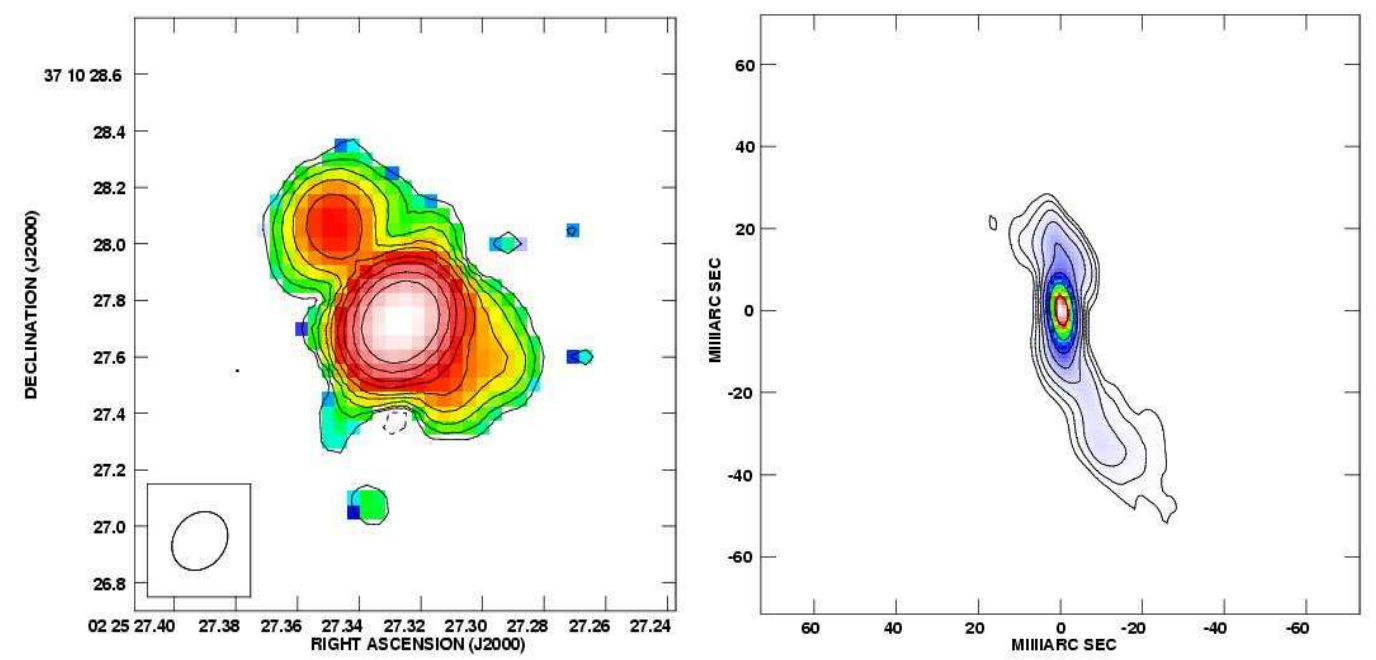

Figure 2: $0222+36$. Left: VLA image at $22 \mathrm{GHz}$ (lowest contour $0.17 \mathrm{mJy} / \mathrm{b}$, peak $89 \mathrm{mJy} / \mathrm{b}$ ), right: VLBI 1.6-GHz image of the core region (1.c. $0.33 \mathrm{mJy} / \mathrm{b}$, p. $61.2 \mathrm{mJy} / \mathrm{b}$ ).

structure of $0222+36$ into a core and two components on either side with an S-shaped structure (see Fig. 1). The phase-referenced VLBA image accounts for $102 \mathrm{mJy}$ of flux density at $1.6 \mathrm{GHz}$ in total intensity. The source is two sided, with jets emerging in opposite directions along the northsouth axis. The unresolved central core has a flux density of $58.2 \mathrm{mJy}$. Both jets have a slightly bent path and become aligned with the kpc-scale main axis at $\sim 20$ mas from the core.

Both the pc- and kpc-scale morphology strongly suggests that the source is oriented near the plane of the sky. At 10 mas from the peak, the jet/counter-jet brightness ratio is $R=B_{\mathrm{J}} / B_{\mathrm{CJ}}=$ $1.3\left(B_{\mathrm{J}}=12.0 \mathrm{mJy}\right.$ and $B_{\mathrm{CJ}}=9.5 \mathrm{mJy}$, with the main jet being the one pointing to the north); this corresponds to $\beta \cos \theta \sim 0.05$ which implies $\theta>85^{\circ}$ if $\beta>0.6$. Therefore, the source is not affected by orientation effects and it has to be intrinsically small. If we consider the largest 

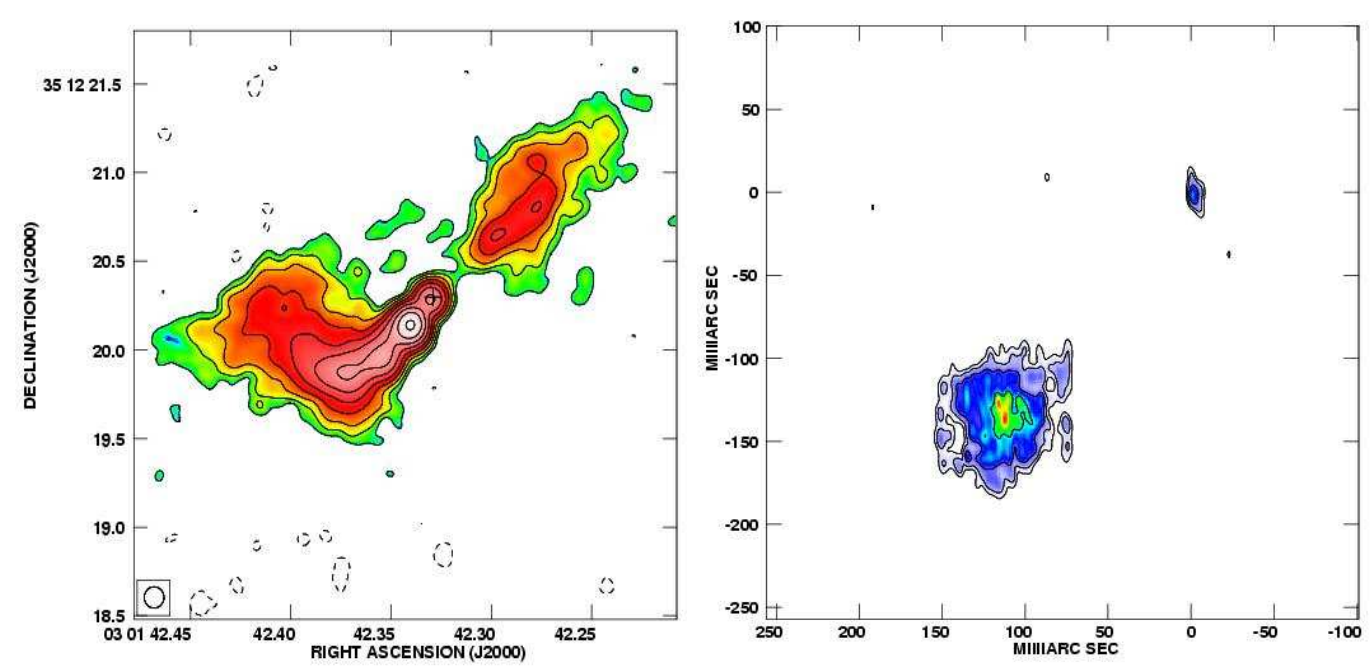

Figure 3: 0258+35. Left: VLA image at $22 \mathrm{GHz}$ (lowest contour $0.19 \mathrm{mJy} / \mathrm{b}$, peak $56 \mathrm{mJy} / \mathrm{b}$ ), right: VLBI 1.6-GHz image of the core and inner jet region (1.c. $0.40 \mathrm{mJy} / \mathrm{b}, \mathrm{p} .7 .5 \mathrm{mJy} / \mathrm{b}$ ).

angular extent of the source and deproject it with an angle of $85^{\circ}$, we derive an intrinsic size of approximately $5.4 \mathrm{kpc}$.

\subsection{VLBI data: $0258+35$}

On kiloparsec scale, this source appears as an interesting FRI-like object, except for that it is only $\sim 5 \mathrm{kpc}$ from tip to tip. There are two prominent compact components in its inner region, and thanks to the phase-referenced VLBI data we can identify these two features: one of them is a 7-mJy compact, flat spectrum $(\alpha=0.0)$ core, whereas the second one has a larger flux density, a steeper spectrum $(\alpha=0.5)$ and is more extended, so it is more likely to be a jet knot, partly resolved. It is worthwhile to stress the importance of the phase-referencing mode, since any attempt to calibrate the source without the solutions from the phase calibrator would have forced all the flux density into one single component (i.e. the core); these would have caused a loss of information about the second component as well as a totally wrong measurement of the core flux density.

\subsection{VLBI data: other sources}

Also in $0648+27$, the phase-referencing is essential for an understanding of its real nature. While it had been proposed that this was a compact symmetric object (CSO) [7], our VLBI observations radically change this view: we detect a 5-mJy compact component on milliarcsecond scale in the northern lobe, with a jet-like extension to the south (see Fig. 4). The source is, therefore, more likely to have jets with low kinetic power rather than being a young object.

$1037+30$ is the only LPC in our sample that is FR II-like, with one hot spot in the eastern lobe; the VLBI data successfully reveal both the central 4-mJy core and the hot spot, which is $\sim 1^{\prime \prime}$ $(\sim 1.7 \mathrm{kpc}$ ) distant and $\sim 60$ mas wide. The presence of the hot spot is suggestive of an ongoing advance in the ISM, so that $1037+30$ could be a young source growing up.

Finally, $1855+37$ is the only one source that has not been detected in our VLBI observations. The rms noise in our field is $0.38 \mathrm{mJy} / \mathrm{beam}$. 

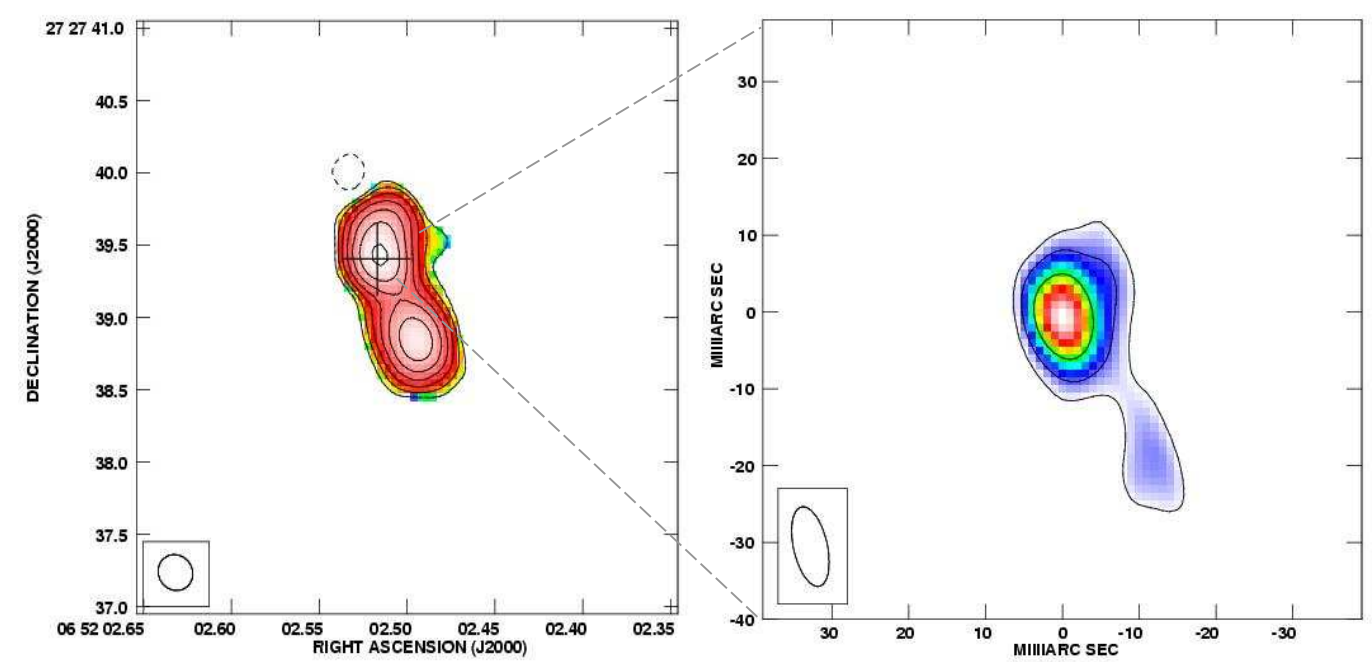

Figure 4: $0648+27$. Left: VLA image at $8 \mathrm{GHz}$ (lowest contour $0.16 \mathrm{mJy} / \mathrm{b}$, peak $11.5 \mathrm{mJy} / \mathrm{b}$ ), right: VLBI 1.6-GHz image of the compact component in the northern lobe (1.c. $0.57 \mathrm{mJy} / \mathrm{b}$, peak $4.4 \mathrm{mJy} / \mathrm{b}$ ).

\subsection{Integrated spectra}

We have also collected integrated spectra adding our new VLA measurements to data dug out from the literature. Typically, this extends the frequency range of the spectra by almost one magnitude, which is vital when it comes to fit synchrotron ageing models. Moreover, our observed VLBI flux densities reassure us that the core contribution is not dominant so we can neglect it and consider the fit to the integrated spectra. We adopt continuous injection models, which reveal break frequencies in the $\mathrm{GHz}$ domain. Under basic assumptions on the magnetic field strength (equipartition conditions), we therefore derive spectral ages in the range $10^{5}-10^{7} \mathrm{yrs}$.

\section{Discussion}

Our high-resolution images resolve the five Low-Power Compact Radio sources we observed. The combined information of flat/inverted spectral indices $\left(-0.2 \leq \alpha_{1.6}^{22} \leq 0.5\right)$ and compactness unambiguously identify radio cores. Most sources are symmetric, at least on arcsecond scales; $0222+36$ is also clearly symmetric on milliarcsecond scales. In general, the objects do not show any evidence for strong beaming effects (e.g. strong, one-sided jets, dominant cores). Thus, these sources are most likely close to the plane of the sky. Their deprojected linear size must be, therefore, within a factor of a few from their observed dimensions. We confirm that this class of radio sources is characterized by a linear size $<10 \mathrm{kpc}$. We note that the small size of LPCs is not related to the low power of the radio source, since there is also a large number of extended (LS $\left.>10^{2} \mathrm{kpc}\right)$ radio galaxies with a similarly low radio power.

However, LPC sources are more extended than classical young radio sources like the CSOs [8], and could represent the intermediate age sources between CSOs and classic kpc-scale radio galaxies. The youth scenario for CSOs has the problem of the lack of objects filling in the gap between sources with age $<10^{4} \mathrm{yrs}$ and $>10^{7} \mathrm{yrs}$; in particular, there seem to be too many bright young sources. However, this problem can be solved if the radio sources dim in luminosity as they 
grow up. Other possible explanations involve a significant fraction of either frustrated or short-lived sources [9].

The detailed study of the compact sources in the BCS is important to investigate these possibilities. If we consider the data presented here for five sources and include also the two other sources previously studied (4C 31.04, NGC 4278 [3, 4]), we are facing a variety of behaviours. As a matter of fact, we have cases where there is clear evidence of a source which is growing, with a significant interaction between the jet and the ISM. On the other hand, we also find sources that are small because they are either short lived or frustrated: the dynamic ages estimated from the large-scale structure and assuming typical lobe advance velocities are typically about one order of magnitude lower than the spectral estimates. Unless there is some misleading assumption (e.g., equipartition conditions do not apply), the advance velocities $(\ll 0.1 c)$ have to be significantly lower than those measured in CSOs.

\section{Four things to remember}

- A substantial fraction of sources in the Bologna Complete Sample have radio powers in the FR I range but significantly smaller linear size, so that they appear compact on arcsecond scale. We have termed these sources Low-Power Compact (LPC) and we have observed five of them at high resolution.

- VLA images resolve the sub-arcsecond structure. VLBI phase referenced observations detect compact cores in 4 out of 5 sources and parsec-scale features (including one hot spot) in three of them. Phase referencing proves to be essential in both detecting the sources and attaining as much as possible information about their structure.

- The objects are not strongly beamed as suggested by the low core dominance and the twosidedness. Therefore, LPCs have to be intrinsically small $(<10 \mathrm{kpc})$ and some of them are good candidates for frustrated or prematurely dying radio sources.

- The study of the BCS, as a well-defined complete sample, will be important to derive the source evolution with a statistical approach.

\section{References}

[1] Giovannini G., these proceedings

[2] Giovannini G. et al. 2005, ApJ, 618, 635

[3] Giroletti M. et al. 2003, A\&A, 399, 889

[4] Giroletti M. et al. 2005, ApJ, 622, 178

[5] Giroletti M. et al. 2005, A\&A, 441, 89

[6] Fanti C. et al. 1986, A\&AS, 65, 145

[7] Morganti R. et al. 2003, A\&A, 399, 511

[8] Gugliucci N.E. et al. 2005, ApJ 622, 136

[9] Stanghellini C., these proceedings 